\title{
Evaluation of The Maxillary Basal Bone and Molar Change of Skeletal Class II Malocclusion Treated by Saggital-Guidance Twin-Block
}

\author{
Yingyu Yi, Weixing Quan* and Long Qian* \\ The $2^{\text {nd }}$ Dental Center, Ninth People's Hospital, Shanghai Jiao Tong University School of Medicine, 280 Mohe Road, Shanghai 201999, China \\ Corresponding author: Weixing Quan, The $2^{\text {nd }}$ Dental Center, Ninth People's Hospital, Shanghai Jiao Tong University School of \\ Medicine, 280 Mohe Road, Shanghai 201999, China \\ Long Qian, The $2^{\text {nd }}$ Dental Center, Ninth People's Hospital, Shanghai Jiao Tong University School of Medicine, 280 Mohe Road, \\ Shanghai 201999, China
}

\section{ARTICLE INFO}

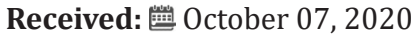

Published: 㠒 October 15, 2020

Citation: Yingyu Yi, Weixing Quan, Long Qian. Evaluation of The Maxillary Basal Bone and Molar Change of Skeletal Class II Malocclusion Treated by SaggitalGuidance Twin-Block. Biomed J Sci \& Tech Res 31(2)-2020. BJSTR. MS.ID.005065.

Keywords: Saggital-guidance Twinblock; Pancherz analysis; Skeletal Class II malocclusion

\section{ABSTRACT}

Objective: To investigate the effects of Saggital-guidance Twin-block appliance(SGTB) on maxillary basal bone and molar with skeletal class II malocclusion.

Methods: The cephalometric datas, including thirty-four skeletal Class II patients with SGTB and non-extraction treatment, was quantitatively analyzed with Pancherz.

Results: After orthopedic treatment, the maxillarretruded $(0.68 \mathrm{~mm}, \mathrm{P}<0.01)$, the upper molar teeth were changed posteriorly $(1.33 \mathrm{~mm}, \mathrm{P}<0.01)$.After orthodontic treatment, the maxillary bone still turned out posterior position $(0.65 \mathrm{~mm}, \mathrm{P}<0.05)$, and the molar teeth slightly retruded $(0.53, \mathrm{P}>0.05)$.

Conclusions: The correction of skeletal Class II malocclusion would be achieved by semi-fixed Twin-block.

\section{Introduction}

Class malocclusion is the most common type of orthodontic malocclusion in clinical orthodontics, and the prevalence of Chinese permanent teeth in the early stage accounts for about $19.41 \%$ to $26.62 \%[1,2]$. It's typically characterized by maxillary anterior process and/or mandibular retraction with deep anterior occlusion and deep coverage of the anterior teeth. Because the convexity of the upper jaw is higher than the lower jaw, the prevalence of patients in China is higher, so The Chinese patients with type II skeletal pattern are mostly clinical manifestations of maxillary anterior process with mandibular retraction. The use of functional appliances for dual phase treatment of osteopathic class II patients in the growth and development stage has been controversial in recent years, but based on the different treatment mechanisms of functional appliances, many scholars still believe that functional appliances can obtain good clinical results. Effect[3,4] at present, functional appliances commonly used for early correction of type II malocclusion include movable appliances such as Activator, Frankel, Bionator, Twin-block, and fixed appliances Herbst, Forsus and so on.

Saggital-guidance Twin-block appliances(SGTB) are an improved semi-adhesive Twin-Block appliance. It consists of two parts: the upper jaw is the first premolar (or first milk molar) that surrounds the upper jaw and extends backward to most of the posterior crowns. The occlusal pad is bonded and fixed to the upper jaw, and the bracket is embedded in the occlusal pad at the center of the first premolar (or first milk molar) of the upper jaw relative to the lip surface of the crown. Adhesion can align the upper anterior teeth with functional correction. If necessary, a spiral expander (Dentaurum standard spiral expander (600-301-30) is placed at the midline position of the maxillary condyle in some cases of 
maxillary dental arch stenosis to expand the width of the upper jaw facilitates the advancement of the lower jaw. The lower jaw is a movable occlusal pad that extends forward from the tip of the second premolar (or second milk molar) of the lower jaw to the second premolar (or second milk molar) on the other side.

The cusp of the tooth and the occlusal cushion of the second premolar (or the second milk molar) and the maxillary $70^{\circ} \sim 45^{\circ}$ staggered relation to the inclined surface, while the cutting head is provided between the card and the adjacent hook retention increases.Saggital-guidance Twin-block has obtained the national practical patent certification No: ZL201320028895.0, which is used to correct bony type II malocclusion, and the related effects on the jaw and occlusal plane have been studied[5-7], However there is no relevant research data on the effect of maxillary bone and maxillary dentition. The purpose of this study is to collect skeletal class II malocclusion patients treated with SGTB appliance using x-ray cephalometry to evaluate SGTB's osteopathic II The effect of maxillary molar relocation and fixed fixtures on maxillary retreat in this type of patients, and the clinical application and development prospects of SGTB are discussed.

\section{Materialsand Methods}

\section{Case Selection}

Collecting growth-stage bone type ii non-extraction cases that have been treated in the Department of Orthodontics, Department of Orthodontics, Ninth People's Hospital, Shanghai Jiaotong University School of Medicine, since 200834 cases have completed all orthodontic treatments.

Case inclusion Criteria:(1)The clinical examination was Antarctic II malocclusion, the bilateral molar relationship was distant, and the anterior teeth covered $\geq 5 \mathrm{~mm}$; (2)X-ray skull lateral radiograph shows ANB angle $\geq 5^{\circ}[8,9]$, The mandibular plane angle is average or low angle $\left(\mathrm{FMA} \leq 32^{\circ}\right.$ ); (3) No tooth extraction during phase ii treatment; (4)According to the cervical vertebrae bone maturity index (CVMIs) to judge the bone age, all patients were in the pre-peak and peak stages of growth and development (S1 S3).

Exclusion Criteria: (1)Have received orthodontic treatment; (2) Temporomandibular disorder (TMD) symptoms; (3)Systemic systemic disease.

\section{SGTB Design and Treatment Procedures}

SGTB Design: The Saggital-guidance Twin-block appliance (SGTB) is an improved semi-adhesive Twin-Block appliance.It consists of two parts: the upper jaw is wrapped around the first premolar (or first milk molar) of the upper jaw and faces The occlusal pad that extends posteriorly to most of the posterior dental crowns is adhesively fixed to the upper jaw, and the bracket is embedded in the occlusal pad at the center of the first premolar (or first milk molar) of the upper jaw relative to the lip surface of the crown. Combined with the fixed bracket bracket of the maxillary anterior teeth, the upper anterior teeth can be aligned at the same time as functional correction. If necessary, in some cases of maxillary arch stenosis, a spiral expander (Dentaurum standard spiral expander 600-301-30), to increase the width of the upper jaw, which is conducive to the advancement of the lower jaw. The lower jaw is a movable occlusal pad that extends forward from the tip of the second premolar (or second milk molar) of the lower jaw to the second front of the other side Molar (or second milk molar) cusp, and the second premolar (or second milk molar) and the maxillary occlusal pad are in a staggered relationship of $70^{\circ} \sim 45^{\circ}$. At the same time, a shear card and an interproximal hook are set to increase the solidity. Bit.

\section{SGTB Treatment Procedure:}

A. Primary Treatment: SGTB orthopedic phase,The upper and lower occlusal occlusal pads of SGTB are fixed on the occlusal surface of the maxillary posterior teeth. The patient cannot remove it by himself. The lower jaw of the appliance is still a movable occlusal pad.The upper and lower occlusal pad guide slopes are standard Twin-block design. The period of orthopedic treatment was maintained for 4 to 13 months (average 10.1 months). When the first molar was neutral and the mandible did not retreat spontaneously, the maxillary occlusal cushion was removed and it entered the second stage of treatment.

B. Second-Stage Treatment: Fixed correction period,The second-stage treatment is mainly to align and level the dentition, establish a neutral molar relationship, and restore and maintain the coordination and beauty of the dentition, jaw, and soft tissue. The treatment time is 10 to 29 months (average 19.8 months).

\section{Cephalometry Analysis}

The time point $\mathrm{T} 0$ is set before the appliance is worn, the time point T1 is after the appliance is deactivated and removed, and the time point is T2 after all fixed corrections are completed. X-ray head lateral positions were taken at the median position of the jaw at 3 time points Positioning film (Kodak 8000c system, United States), combined with common cephalometric measurements and Pancherz measurement and analysis method, using spss 20.0 statistical software to compare the effects of T0 stage and T1 stage, T0 stage and T2 stage on maxillary molar distal displacement and maxillary recession.

\section{Marking Points and Datum Planes (Figure 1)}

In Figure 1, X-ray Cephalometric mark point and datum plane: (1) Saddle point (s) - the midpoint of the saddle image; (2) Nasal point(n) - foremost point of nasal forehead suture; (3)SN plane - a line passing through points $\mathrm{s}$ and $\mathrm{n}$, representing the stable anatomical structure of the skull base, as an overlapping plane; (4) Mesial contact point of the maxillary first molar (M6) - the highest point of the mesial surface of the maxillary first molar; 
(5) Upper alveolar seat point (A) - the most concave point of the bone between the anterior nose spine and the upper alveolar edge point;(6) Upper alveolar edge point (SPr) - the front most lower point of the upper alveolar groove; (7) Upper incisor incisor (U1) the foremost incisor of the upper incisor; (8)Fore-nose spine (ANS) - the tip of the fore-nose spine; 9Posterior nasal spine (PNS) - the tip of the posterior bone spine of the hard palate; (10)Palatal plane (PP) - the connection between the posterior nasal spine and the anterior nasal spine. It represents the stable anatomical structure of the maxilla as a reference line; (11)Vertical reference line (reference line perpendicular) - Vertical reference plane perpendicular to RL through S point.

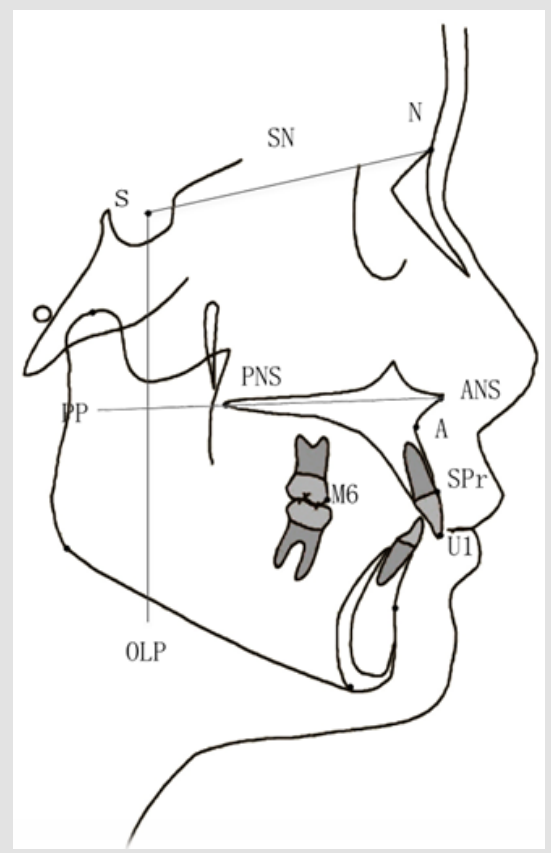

Figure 1: Marking Points and Datum Planes

\section{Measurement Items (Figure 2)}

In Figure 2, Pancherz analysis[10]: Take the sacral plane (PP) on the skull lateral radiograph before treatment and the perpendicular line olp of the sacral plane (the $S$ point is the vertical line of the sacral plane) as the coordinates, with the $S$ point as the overlapping point, and overlapping the SN line, Transfer to the head image after treatment. (1)M6-OLP - distance from the maxillary first molar to the vertical reference plane, which represents the sagittal upward position of the maxillary molar.2(U1-OLP - the distance from the incisal edge of the upper incisor to the vertical reference plane, representing the position of the maxillary incisor upward.(3)AOLP - the distance from the upper alveolar seat point to the vertical reference plane, representing the position of the maxillary basa bone sagittal. (4)SPr-OLP - the distance from the upper alveolar edge point to the vertical reference plane, which can be combined with U1-OLP and A-OLP to represent the amount of change in the rotation of the upper central incisor.

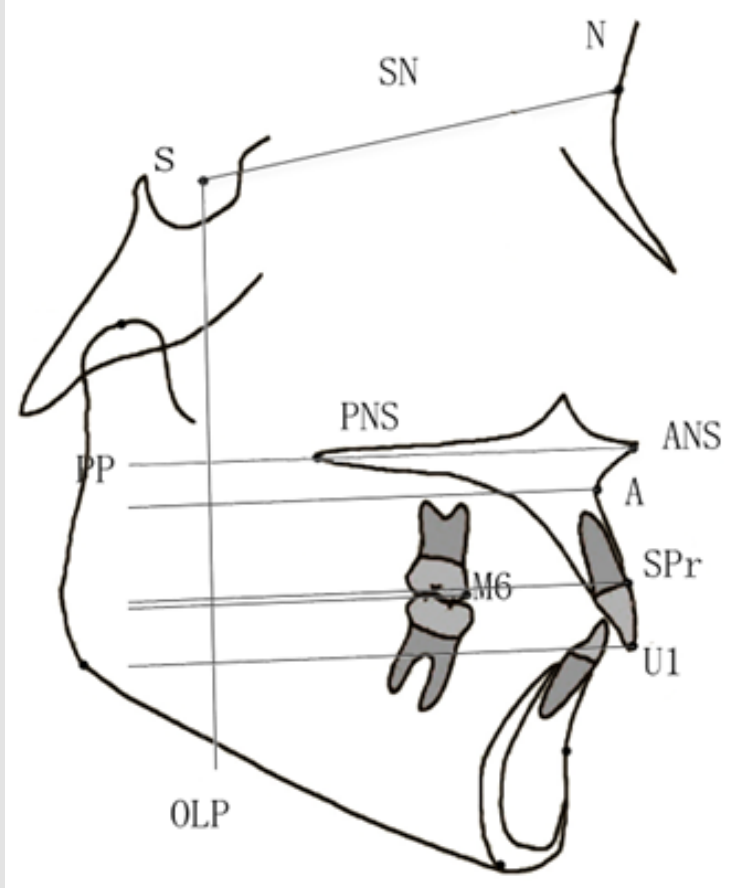

Figure 2: Measurement Items.

\section{Data Processing and Statistical Analysis}

Measurement Error Check: In order to ensure the reliability of the tracing measurement data, the authors used OnyxCeph 2.6 cephalometric measurement analysis software (Image Instruments, Germany) to perform all the fixed points and measurements on the digital lateral film for a continuous period of time. About one week apart, under the same conditions Make the second set point and measurement and perform the paired t test on the results of the two measurements. After the test has no significant difference, take the average value as the final measurement result[11]. The measurement error is calculated by randomly selecting 20 pieces from all the lateral films, Import the difference between the two measurement results into the formula: $S e=\sqrt{ } \sum \mathrm{d} 2 / 2 \mathrm{n}$.

Statistical Analysis: The measurement data were analyzed by SPSS 20.0 software package, and the difference before and after treatment was analyzed by paired test. $\mathrm{P}<0.05$ was statistically significant.

\section{Result}

\section{Test Results of Measurement Errors}

The paired T test was used for the two measurement results, and the P value was 0.217 , which was greater than 0.05 . There was no statistical difference between the two measurement results, so the average value was taken. After measurement and analysis, the measurement error was $0.09 \sim 0.16 \mathrm{~mm}$. It indicates that the data is reliable, that is, the measurement Errors are negligible in this study. 


\section{Comparison of Cephalometric Measurements and their Differences}

Changes in the maxilla and mandible: After SGTB treatment, SNB increases, ANB decreases, ANS-PNS increases, Ar-Go increases, Go-Me increases, S-Go increases, N-Me increases, and APDI increases, N-ANS increased, Me-ANS increased, ODI decreased, and $\mathrm{N}$-ANS/Me-ANS decreased, and the differences were statistically significant. Changes in upper and lower dentition: After SGTB treatment, U1/PP decreased, U6/PP decreased, L1/MP increase, OB decrease, and 0J decrease were statistically significant, while U1/ L1 and L6/MP increased to some extent, but the differences before and after treatment were not statistically significant.After the end of SGTB treatment, the maxillary first molars moved distantly $(1.33 \mathrm{~mm})$, and the maxillary central incisors moved backwards $(1.48 \mathrm{~mm})$ with the growth and development of the entire upper jaw inhibited or even moved backwards $(0.68 \mathrm{~mm})$, and the first stage The differences before and after orthopedic treatment were statistically significant $(\mathrm{P}<0.01)$. After the completion of the orthodontic treatment, compared with before treatment, the maxillary first molar moved $0.53 \mathrm{~mm}$ distally, the position of the upper central incisor moved backward $1.37 \mathrm{~mm}$, and the position of the upper alveolar socket moved backward $0.65 \mathrm{~mm}$, of which the upper central incisor and upper teeth The variable at the slot seat point was statistically significant $(\mathrm{P}<0.05)$ (Tables 1 \& 2).

Table 1: Pancherz head shadow measurement items before and after SGTB appliance treatment ( $\mathrm{mm}$, mean $\pm \mathrm{SD})$.

\begin{tabular}{|c|c|c|c|c|}
\hline $\begin{array}{c}\text { Measurement } \\
\text { item }\end{array}$ & M6-OLP & U1-OLP & A-OLP & SPr-OLP \\
\hline T0 & $36.48 \pm 3.74$ & $67.03 \pm 6.19$ & $61.32 \pm 4.40$ & $64.64 \pm 4.85$ \\
\hline T1 & $35.14 \pm 3.29$ & $65.55 \pm 5.03$ & $60.64 \pm 4.39$ & $63.80 \pm 4.61$ \\
\hline T2 & $35.94 \pm 3.79$ & $65.66 \pm 4.70$ & $60.66 \pm 4.01$ & $64.26 \pm 4.34$ \\
\hline
\end{tabular}

Table 2: Differences in maxillary molars and maxillary bones before and after treatment $(\mathrm{mm}$, mean $\pm \mathrm{SD})$.

\begin{tabular}{|c|c|c|c|c|}
\hline $\begin{array}{c}\text { Measurement } \\
\text { item }\end{array}$ & M6-OLP & U1-OLP & A-OLP & SPr-OLP \\
\hline T1-T0 & $-1.33 \pm 1.22^{* *}$ & $-1.48 \pm 2.19 * *$ & $-0.68 \pm 0.94 * *$ & $-0.83 \pm 1.34 * *$ \\
\hline T2-T0 & $-0.53 \pm 2.22$ & $-1.37 \pm 3.24 *$ & $-0.65 \pm 1.69 *$ & $-0.38 \pm 2.11$ \\
\hline & & $* *: \mathrm{P}<0.01$ & $*: \mathrm{P}<0.05$ & 无 $: \mathrm{P} \geq 0.05$ \\
\hline
\end{tabular}

\section{Discuss}

SGTB is a semi-adhesive Twin-block, It uses the contact of the inclined surface during occlusion to generate continuous proprioceptive stimuli, which affects the morphology and structure of supporting bone and trabeculae, and the relative position of the upper and lower jaws[12]. In recent years, many scholars have studied the orthopedic effects of traditional Twin-Block appliances, mainly focusing on changes in the position and growth of the mandible. Based on the observations of a large number of clinical cases, SGTB not only has an orthopedic effect on the mandible, but also affects the upper jaw. The role of molars and maxilla cannot be
ignored.In this study, the correction of maxillary sagittal direction of patients with type II malocclusion by SGTB was obtained by Pancherz analysis.The maxillary molars were moved farther and the basal bone was moved backwards. Pancherz analysis was established directly by the OLP reference line. Measure changes in the maxillary bones and teeth in the sagittal direction[13], This is the basis for selecting this study to evaluate the effect of sgtb appliance on maxillary molars and maxillary sagittal correction.

Observed from clinical cases, 8 to 10 months after SGTB treatment, most patients have a gap of 2 to $3 \mathrm{~mm}$ between the premolar and the canine. Because the upper jaw of the SGTB uses the premolar and the molar as a whole, the Functional potential energy, which is transmitted to the upper jaw, squeezes the bone gap, and pushes the maxillary molars backwards $(\mathrm{P}<0.01)$. The back of the maxillary molars can establish a stable cuspidial relationship with the mandibular forward molars. Difficulty of type II mismatch treatment; on the other hand, SGTB has improved bracket bonding on the part of the maxillary first premolar, and with the alignment of the anterior segment at the end of the orthopedic stage, the position of the upper anterior tooth can also be moved backward $(\mathrm{P}<0.01)$. At the same time, in the later stage of correction, the application of implant anchorage also reduces the anterior teeth coverage, inhibits the maxillary bone from growing forward, and even pushes the entire maxillary bone backward $(P<0.05)$, and this kind of basal bone There was still a statistically significant difference in postpositional shift after the entire treatment $(\mathrm{P}<0.05)$.

The object of this study is mainly patients at the peak of growth and development. Due to the constraints of medical ethics and realistic conditions, patients with natural growth of type ? malocclusion cannot be used as a control group, so the standard will be included in the phase II treatment. For patients who have not extracted, the effects of extraction on maxillary molars and maxillary bone movements are excluded, and the changes in dental and bony properties of $\mathrm{T} 2$ and $\mathrm{T} 0$ and $\mathrm{T} 1$ and $\mathrm{T} 0$, respectively, are found, although with the eruption of the second molar and the maxillary forward Natural downward growth.Although the molar has moved back after the end of phase II treatment, there is no statistical difference in this variable, but the change in the position of the basal bone is still statistically significant after the end of the treatment, which indirectly proves that sgtb affects molars. The change of relationship is not only a result, but also a means; it not only inhibits the growth of the maxilla, but also achieves the effect of pushing the maxilla backward, thereby changing the sagittal position of the maxillary base and improving type II errors. The problem of occlusal deformity is difficult to solve the problem of sagittal irregularity of the jaw.

It is worth noting that, in combination with the amount of back movement of the upper incisor margin point (SPr), compared with the amount of change in the upper incisor margin (U1) and the upper alveolar seat (A), it was found that The incisor's backward 
movement is mainly inclined. Although it is beneficial to reduce the anterior teeth coverage, it is a burden on the type II deep overbite, which indicates that the SGTB mandibular device must not only guide the mandible, but also To rotate the mandible clockwise while opening the occlusion, the mandibular plane angle increases, so the SGTB appliance is more suitable for all low-angle bone type II cases.

\section{References}

1. Fu minkui, Zhang Ding, Wang bangkang (2002) Investigation on the prevalence of malocclusion in 25392 children and adolescents in China Chinese Journal of Stomatology 37 (5): 371-373.

2. Fu minkui (2003) Orthodontics. Beijing: People's Health Press.

3. Fu minkui (2007) Special course of Orthodontics. Beijing: People's Health Press.

4. Law JW, Hagg U (1999) Cephalometric morphology of Chinese with Class II division 1 malocclusion[J]. Br Dent J 186(4): 188-190.

5. Li Xingui, ye Jinmei, Zhang Dongjie (2014) Pitchfork analysis to evaluate the hard tissue efficacy of twin block appliance in the treatment of angle II malocclusion. Chinese Journal of clinicians 8 (14): 2634-2639.

6. Wang Xi, Zuo Yanping, Dong Fusheng. (2013) Pancherz analysis to evaluate the hard tissue effect of twin block appliance in the treatment

\section{ISSN: 2574-1241}

DOI: $10.26717 /$ BJSTR.2020.31.005065

Weixing Quan, Long Qian. Biomed J Sci \& Tech Res

(C) This work is licensed under Creative

Submission Link: https://biomedres.us/submit-manuscript.php of juvenile angle II malocclusion. Chinese Journal of practical dentistry 6 (3): 153-157.

7. Sheng Haijing (2012) Clinical study of twin block functional appliance in the treatment of Angle Class II class I malocclusion. Stomatology 32 (6): 357-358

8. Pancherz H, Hansen K (1986) Occlusal changes during and after Herbst treatment: a cephalometric investigation[J]. Eur J Orthod 8(4): 215-228.

9. von Bremen J, Pancherz H (2005) Association between Björk's structural signs of mandibular growth rotation and skeletofacialmorphology. Angle Orthod 75(4): 506-509.

10. Tewson DH, Heath JK, Meikle MC (1988) Biochemical and autoradiographical evidence that anterior mandibular displacement in the young growing rat dose not stimulatecell proliferation or matrix formation at the mandibular condyle. Arch Oral Biol 33(2): 99-107.

11. Peng Jing, Deng Hui, Cao Caifang (2001) Longitudinal study on the depth change of craniomaxillary complex in 7-12-year-old female children. Orthodontics 4.

12. Shen (2005) The role of type X collagen in facilitating and regulating endochondral ossification of articular cartilage[J]. Orthod Craniofac Res 8(1): 11-17.

13. Pancherz HA (1984) Cephalometric analysis of skeletal and dental changes contributing to Class II correction in activator treatment[J]. Am J Orthod 85(2): 125-134.

$\begin{array}{ll}\text { BIOMEDICAL } & \text { Assets of Publishing with us } \\ \text { RESEARCHES } & \text { - Global archiving of articles } \\ & \text { - Immediate, unrestricted online access } \\ & \text { - Rigorous Peer Review Process } \\ & \end{array}$

